\title{
Redox Factor-1 Mediates Inflammatory Response during Tumor Promotion in Skin Epidermal JB6 Cells
}

\author{
Delira Robbins ${ }^{1}$, Wenjuan $\mathrm{Li}^{1}$, Katie Humphrey ${ }^{2}$, Yunfeng Zhao ${ }^{{ }^{*}}$ \\ ${ }^{1}$ Department of Pharmacology, Toxicology \& Neuroscience, Louisiana State University Health Sciences Center, \\ Shreveport, USA \\ ${ }^{2}$ Southwood High School, Shreveport, USA \\ Email: yzhao1@1suhsc.edu
}

Received August 20, 2012; revised September 30, 2012; accepted October 19, 2012

\begin{abstract}
Recently, redox factor-1 (Ref-1) has received considerable attention as an enzyme for stimulating tumor cell growth. We hypothesized that Ref-1 is upregulated during the early stage of tumorigenesis. Utilizing both tumor promotion sensitive $\mathrm{P}+$ and promotion resistant $\mathrm{P}-$ mouse skin epidermal JB6 cells, we found that Ref-1 expression was only induced in tumor promotion sensitive P+ cells following TPA treatment. Consistent with that, Ref-1 knockdown suppressed skin cell transformation. Interestingly, Ref-1 knockdown suppressed nuclear translocation of NF-kappaB subunit p65, and inhibited production of proinflammatory cytokines. These results suggest Ref-1 may promote early tumorigenesis and serve as a target for chemoprevention.
\end{abstract}

Keywords: Ref-1; Tumorigenesis; JB6 Cells; Tumor Promotion; Chemoprevention

\section{Introduction}

Apurinic/apyrimidinic (AP) sites can potentially block DNA replication and are cytotoxic and mutagenic to cells $[1,2]$. Human apurinic (apyrimidinic) endonuclease 1 (APE1) plays a key role in the base excision repair pathway (BER), and is a major repair protein for abasic sites [3]. APE1 was later coined redox factor-1 (Ref-1) due to its ability to sense changes in the intracellular redox status and activate transcription factors of the stress response pathway. APE1/Ref-1 is a ubiquitously expressed multifunctional protein also known to regulate the tranpscription of stress inducible genes such as NF- $\kappa \mathrm{B}, \mathrm{p} 53$, HIF- $1 \alpha$ and AP-1.

APE1/Ref1 expression levels have been found to be elevated in a number of cancers such as ovarian, cervical, prostate, rhabdomyosarcoma and germ cell tumors and correlated with the radiosensitivity of cervical cancers [4]. It has been suggested that APE1/Ref-1 provides an eloquent link between cancer, DNA repair, transcription factor regulation, oxidative signaling and cell cycle control which suggest APE1/Ref-1 as a potential chemopreventive target in tumorigenesis. In order to determine the role of Ref-1 in tumor promotion, we used tumor promotion-sensitive JB6 $\mathrm{P}+$ and promotion-resistant JB6 $\mathrm{P}-$ mouse skin epidermal cells to observe the differential expression of APE1/Ref-1 following TPA treatment, and

${ }^{*}$ Corresponding author. to determine how Ref-1 plays its role in early tumorigenesis.

\section{Materials and Methods}

\subsection{Cell line, Reagents, Treatment}

The murine skin epidermal tumor promotion sensitive JB6 $\mathrm{P}+$ and promotion resistant JB6 $\mathrm{P}-$ cells were purchased from American Type Culture Collection (ATCC, Rockville, MD). Cells were grown in EMEM medium containing $4 \%$ fetal bovine serum (Hyclone), $2 \mathrm{mM}$ L-glutamine (Invitrogen), and $50 \mu \mathrm{g} / \mathrm{ml}$ penicillin/streptomycin (Invitrogen) in a $37^{\circ} \mathrm{C}$ incubator under $5 \% \mathrm{CO}_{2}$. The tumor promoter phorbol ester, 12-O-tetradecanoylphorbol-13-aceteate (TPA; Sigma) was prepared as a 100 $\mathrm{mM}$ stock solution and directly diluted in cell culture medium, with the final concentration being $100 \mathrm{nM}$.

\subsection{Total Cell Lysate}

Collected JB6 P+ and P- cells were suspended in $250 \mu \mathrm{l}$ of phosphate buffered saline (PBS, $\mathrm{pH}$ 7.4) containing a proteinase inhibitor cocktail (Calbiochem). The JB6 cells were collected by centrifugation and resuspended in RIPA buffer (50 mM Tris, $150 \mathrm{mM} \mathrm{NaCl}, 0.1 \%$ SDS, $0.5 \% \mathrm{Na}$. deoxycholate and $1 \%$ Triton $\mathrm{X}-100)$ supplemented with the proteinase cocktail $(5 \mu \mathrm{g} / \mathrm{ml}$ each of pepstatin, leupeptin and aprotinin). Cells were sonicated on ice for two strokes (10 sec per stroke) using a Fisher 
Sonic Dismembrator (Model 100, Scale 4). After incubating on ice for $30 \mathrm{~min}$, cell lysate was centrifuged at $18,000 \times \mathrm{g}$ for $20 \mathrm{~min}$, and the supernatant was collected and designated as Total Cell Lysate.

\subsection{Western Blot Analysis}

Protein concentrations of the samples were determined using a colorimetric assay (BioRad Laboratories). Thirty micrograms of total cell lysate were separated on $10 \%$ SDS-PAGE gels. Proteins were then transferred to nitrocellulose membranes. The membranes were incubated against p65, Ref-1, $\beta$-actin, and SDHB proteins (Santa Cruz Biotechnology) to obtain the results.

\subsection{Ref-1 siRNA Transfection}

Cells were seeded at $2 \times 10^{5}$ cells per well in six-well tissue culture plates. The cells were incubated at $37^{\circ} \mathrm{C}$ in a $5 \% \mathrm{CO}_{2}$ incubator until they became $70 \%-80 \%$ confluent. For each transfection, $3 \mu$ of the Ref- 1 siRNA duplex (Santa Cruz Biotechnology,) were diluted into 100 $\mu l$ of siRNA transfection medium (sc-36868, Santa Cruz Biotechnology) and labeled as Solution A. Solution B consisted of $6 \mu \mathrm{l}$ of transfection reagent (Santa Cruz Biotechnology) diluted into $100 \mu$ of siRNA transfection medium. Solution A and B were mixed gently and incubated for 30 minutes at room temperature. The cells were washed once with $2 \mathrm{ml}$ of siRNA transfection medium. For each transfection, $0.8 \mathrm{ml}$ of siRNA transfection medium were added to each tube containing the solution $\mathrm{A} / \mathrm{B}$ mixture, mixed and directly added to the washed cells. Cells were incubated for 24 hours at $37^{\circ} \mathrm{C}$ in a $5 \%$ $\mathrm{CO}_{2}$ incubator. Immediately following, the transfection mixture was removed and replaced with $2 \mathrm{ml}$ of $1 \times$ normal growth medium. The cells were incubated for an additional $24 \mathrm{~h}$ and assayed via Western blot analysis. Fluorescein conjugated control siRNA (Santa Cruz Biotechnology) was used to monitor transfection efficiency.

\subsection{Soft Agar Assay}

The soft agar cell transformation assay was carried out in six-well plates. The bottom agar was made using $1.25 \%$ agar, $2 \times$ EMEM medium, 10\% FBS, PBS, glutamine, and penicillin and was incubated in a hot water bath for $15 \mathrm{~min}$. The mix was then divided and treated with various treatments. In each well, $3.5 \mathrm{~mL}$ of the agar mix was added and allowed to harden for $30 \mathrm{~min}$. The top agar mix contained $0.5 \%$ bottom agar mix and $2 \times 10^{5}$ of cells. The cell treatments were added at $2 \times$ concentration to the top agar mix and $1 \mathrm{ml}$ of each was added to each well. The agar was allowed to solidify and incubated in a $37^{\circ} \mathrm{C}$ incubator under $5 \% \mathrm{CO}_{2}$ for $14 \mathrm{~d}$. Cells were stained with neutral red dye $(0.25 \mathrm{mg} / \mathrm{ml})$ 2-(p-Iodophenyl)-3- (p-nitrophenyl)-5-phenyl tetrazolium chloride hydrate (Aldrich \#I-1,040-6) containing $1 \mathrm{~mL}$ per well. The dye was sonicated before added to cells. Cells were allowed to stain for twenty-four hours.

\subsection{Quantification of Cytokine and Chemokine Proteins}

JB6 $\mathrm{P}+$ cells were transfected with siRNA to Ref-1 as previously mentioned. Cells were collected and lysed using RIPA buffer to isolate the total cell lysate as previously mentioned. One hundred micrograms of each cell lysate was used for cytokine and chemokine detection and quantification by the Quantibody Mouse Cytokine Array 1 kit (RayBiotech, Inc.) according to the manufacturer's protocol. The signal (Cy3) was captured using an Axon Genepix laser scanner (The Research Core Facility at Louisiana State University Health Sciences Center, Shreveport, LA). Quantitative data and statistical analysis were performed using Prism 3.0 software.

\subsection{Statistical Analysis}

One-way ANOVA followed by the Newman-Keuls posttest were used for multi-group comparisons. Experiments were repeated at least three times. Data were reported as mean \pm standard error (S.E.M.) $\mathrm{p}<0.05$ was considered statistically significant.

\section{Results}

\subsection{Ref-1 Expression Was Only Induced in Promotable JB6 P+ cells Following TPA Treatment}

We first tested whether the tumor promoting phorbol ester, TPA, could induce Ref-1 expression in mouse skin epidermal tumor promotion sensitive $\mathrm{P}+$ and tumor promotion resistant $\mathrm{P}-$ cells. JB6 $\mathrm{P}+$ and $\mathrm{P}-$ cells were treated with TPA (100 nM) for 24 hours. Ref-1 expression was assessed via Western blot analysis. Our results (Figure 1) demonstrated that Ref-1 expression was only induced in JB6 P+ cells compared to JB6 $\mathrm{P}-$ cells as early as $24 \mathrm{~h}$ post TPA treatment.

\subsection{Ref-1 Knockdown Suppressed TPA-Induced Skin Cell Transformation in Tumor Promotion-Sensitive Jb6 P+ Cells via Suppression of TPA-Induced P65 Nuclear Translocation}

To determine whether APE1/Ref-1 plays an important role in skin cell transformation, we knocked down APE1/Ref-1 utilizing siRNA and observed the effects of APE1/Ref-1 knockdown on anchorage-independent growth in soft agar. We found that knockdown of APE1/ 
Ref-1 expression significantly decreased TPA-induced skin cell transformation (Figure 2).

Ref-1 is known as a redox-sensitive upstream regulator of NF- $\kappa \mathrm{B}$. We investigated the effect of TPA-induced Ref-1 expression on p65 expression, the transactivational subunit of NF- $\kappa \mathrm{B}$.

Similar to Ref-1 expression, we observed higher levels of p65 protein expression in JB6 $\mathrm{P}+$ cells compared to $\mathrm{P}-$ cells. In Figure 3, we labeled phosphorylated p65 (Ser 536) with FITC staining and stained the nucleus with DAPI to detect p65 nuclear translocation induced by TPA treatment. To further reveal the potential mechanism of Ref-1 involvement in early stage TPA-induced tumor promotion, we knocked down Ref-1 expression via siRNA and investigated its effect on phosphorylated p65 TPA-induced nuclear translocation. We found following TPA $24 \mathrm{~h}$ treatment that phosphorylated p65 (Ser536) translocated to the nucleus. Interestingly, Ref-1 knockdown suppressed phosphorylated p65 nuclear translocation.

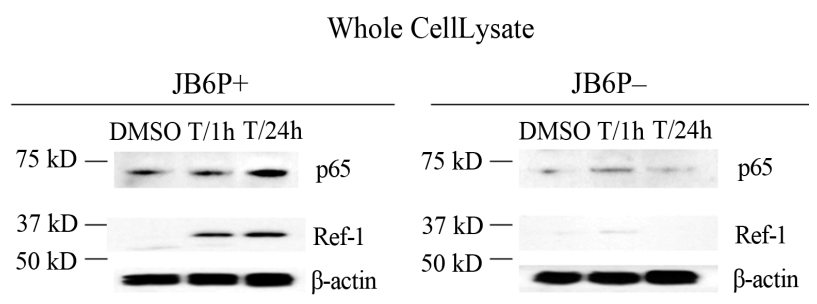

Figure 1. TPA-induced Ref-1 activation was higher in promotable JB6 P+ cells compared to non-promotable JB6 Pcells. JB6 P+ and P-cells were treated with TPA (100 nM) for 1 or $24 \mathrm{~h}(\mathrm{~T} / \mathrm{h} ; \mathrm{T} / 24 \mathrm{~h})$. Whole cell lysate was prepared for the experiments. DMSO: 0.1\% DMSO for $24 \mathrm{~h}$. Western blot analysis of Ref-1, and p65 protein expression. $\beta$-actin, was used as a loading control. Experiments were repeated three times and a representative result is shown.
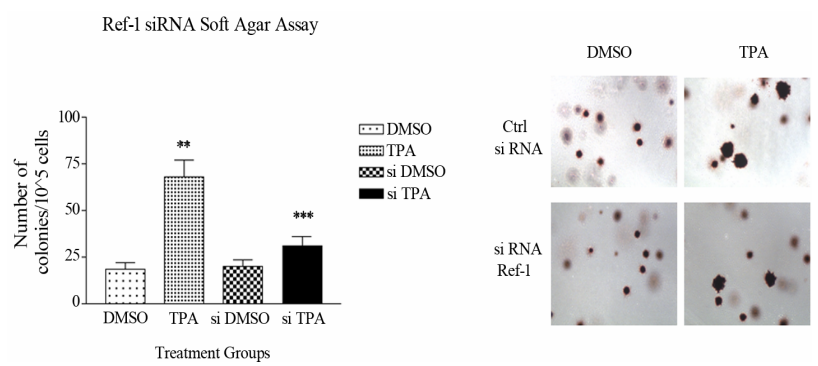

Figure 2. Knockdown of Ref-1 suppressed TPA-induced skin cell transformation. (A) Soft agar colony formation assay was performed using siRNA-transfected cells. (B) Quantification of soft agar assay. siDMSO: siRNA Ref-1 transfected cells treated with $0.1 \%$ DMSO; siTPA: siRNA Ref-1 transfected cells treated with TPA (5 nM). * $\mathbf{p}<0.05$ significantly different from DMSO treatment group; ** $\mathbf{p}<$ 0.05. significantly different from TPA treatment group. Experiments were repeated three times and a representative result is shown.

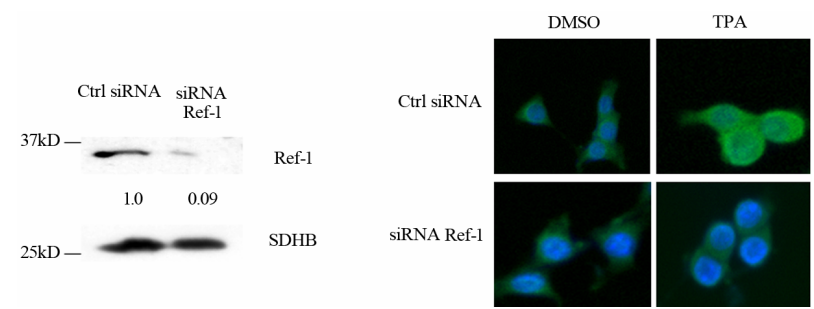

Figure 3. Knockdown of Ref-1 suppressed TPA-induced phosphorylated p65 nuclear translocation. JB6 P+ cells were treated with TPA (100 $\mathrm{nM})$ for $24 \mathrm{~h}$. (A) Western blot analysis of Ref-1 siRNA transfection. (B) Immunofluorescence of JB6 P+ cells. FITC green (phosphorylated p65); DAPI (nuclear staining). Experiments were repeated three times and a representative result is shown. 20X magnification.

\subsection{Ref-1 Knockdown Suppressed Cytokine Expression in Tumor Promotion-Sensitive JB6 P+ Cells}

Previous reports that investigated the influence of inflammation on carcinogenesis were concerned with the early stages of tumor development (i.e. tumor initiation and promotion). In addition, the pathogenesis of tumor development has been generally thought to occur either intrinsically through genetic alterations or extrinsically through external factors to stimulate tumor cells to release inflammatory mediators [5]. To gain further insight into how APE1/Ref-1 may affect early stage tumor promotion, we progressed forward by assessing the effects of APE1/Ref-1 knockdown on NF- $\kappa$ B-mediated cytokine/chemokine expression following TPA treatment in tumor promotion-sensitive JB6 $\mathrm{P}+$ cells. Interestingly, we found that TPA treatment induced an inflammatory response of pro-inflammatory cytokines/chemokines, mainly those involved in leukocyte infiltration. In Figure 4, TPA $24 \mathrm{~h}$ treatment significantly increased $\mathrm{KC}$, monocyte chemotactic protein-1 (MCP-1) and Regulated upon Activation, Normal T-cell Expressed and Secreted (RAN TES), IL-3 and IL-4. KC, also known as CXC1, is secreted by human melanoma cells and has mitogenic properties. This cytokine is a main attractor of neutronphils and expression is mediated by NF- $\kappa \mathrm{B}$ in mice [6]. MCP-1 expression has been observed in various inflamematory diseases [7-9] and tumors [10]. This chemokine is directly induced by TPA treatment and plays a role in the chemotaxis of macrophages [11]. Lastly RANTES, is a chemokine for $\mathrm{T}$ cells, eosinophils and basophils and plays an active role in reactive oxygen species producntion and recruitment of leukocytes to inflammatory sites [12]. Interestingly, knockdown of Ref-1 in both DMSO and TPA treated groups significantly suppressed the expression of these cytokines/chemokines. Nevertheless, knockdown of Ref-1 significantly suppressed expression of both proinflammatory cytokines/chemokines and decreased anchorage-independent growth of JB6 P+ cells. 

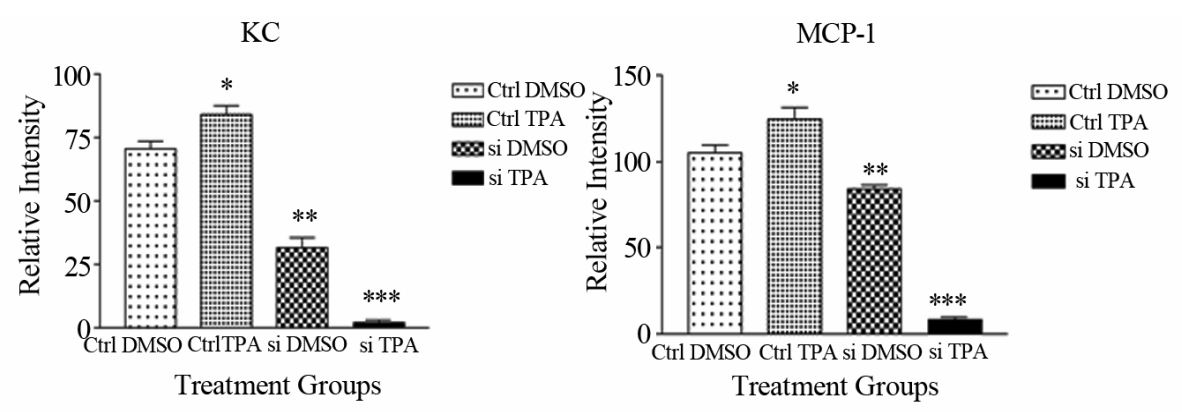

RANTES

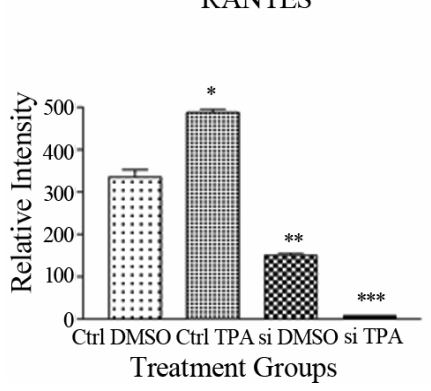

IL-3

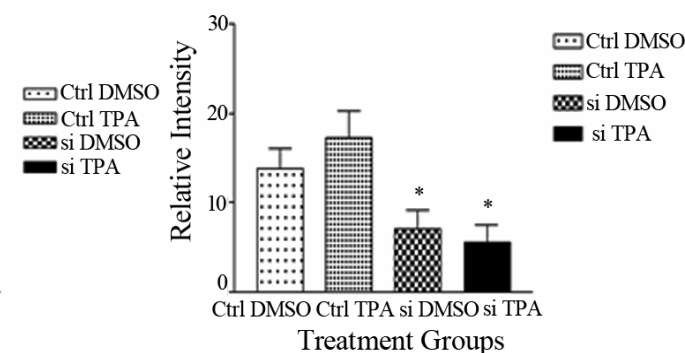

IL-4

Figure 4. Knockdown of Ref-1 suppressed TPA-induced cytokine release in tumor promotable JB6 P+ cells. Quantification of pro-inflammatory cytokines/chemokines. siDMSO: siRNA Ref-1 transfected cells treated with 0.1\% DMSO; siTPA: siRNA Ref-1 transfected cells treated with TPA $(5 \mathrm{nM})$. " $\mathrm{p}<0.05$ significantly different from DMSO treatment group; ${ }^{* * *} \mathrm{p}<0.05$ significantly different from TPA treatment group. Experiments were repeated at least three times and a representative result is shown.

\section{Discussion}

APE1/Ref-1 is ubiquitously expressed in cells with cell type specific cellular localization [13]. In addition, both expression and subcellular localization of APE1/Ref-1 are altered in hyperproliferative disorders such as aging and tumors. It has also been suggested that APE1/Ref-1 expression is inversely correlated with apoptosis suggesting that APE1/Ref-1 may contribute to early stage tumor promotion. We chose to assess the redox activity of APE1/Ref-1 in modulating NF- $\kappa \mathrm{B}$ nuclear translocation and its effect on cytokine release and skin cell transformation. We utilized the murine JB6 skin epidermal cell system to determine the role of APE1/Ref-1 in early stage tumorigenesis and whether APE $1 / \operatorname{Ref}-1$ is a potential novel target for chemoprevention. The mouse Balb/C JB6 cells [14] are the only well-characterized model to study tumor promotion. JB6 cells have two clone variants: tumor promotion sensitive $\mathrm{P}+$ cells and promotion resistant $\mathrm{P}-$ cells. Herein, we found that tumor promotion sensitive JB6 P+ cells expressed higher APE1/ Ref-1 protein levels following TPA treatment which correlates with the current literature that suggests that APE1/Ref-1 expression is higher in cancer cells compared to their normal counterparts $[15,16]$.

$\mathrm{NF}-\kappa \mathrm{B}$ is a known transcription factor that is redox regulated by APE1/Ref-1 through reduction of the criti- cal cysteine residues of NF- $\kappa \mathrm{B}$ [17], we further investigated the correlation of APE1/Ref- 1 and NF- $\kappa$ B expression in early stage skin tumor promotion by comparing the expression of the NF- $\kappa \mathrm{B}$ transactivation subunit $\mathrm{p} 65$ in both promotion-sensitive $(\mathrm{P}+)$ and promotion-resistant $(\mathrm{P}-)$ cells. Elevated levels of p65 following TPA treatment were consistent with elevated levels of APE1/Ref-1 that occurred only in promotion-sensitive $\mathrm{P}+$ cells, suggesting that APE1/Ref-1 and NF- $\kappa \mathrm{B}$ are involved in early stage tumor promotion in skin carcinogenesis. We demonstrated that targeting APE1/Ref-1 in early stage tumor promotion can have direct effects on proinflammatory signaling and skin cell transformation. In addition, knockdown of APE1/Ref-1 significantly reduced the expression of cytokines/chemokines and the colony forming ability of promotion-sensitive $(\mathrm{P}+)$ cells following TPA treatment. There are two common possibilities to explain the differential expression of APE1/Ref-1 in promotion-sensitive $(\mathrm{P}+)$ and promotion-resistant $(\mathrm{P}-)$ cells and the ability of APE1/Ref-1 knockdown to decrease TPA-induced colony formation: 1) the current antioxidant status of the cell type; 2) a decrease in the stress response. Our previous studies have shown that chemoprevention via an antioxidant approach is a novel yet practical strategy to suppress early stage skin carcinogenesis [18]. In addition, we have shown that promotion-resistant $\mathrm{P}-$ cells have higher MnSOD expression 
and activity when compared with promotion-sensitive $\mathrm{P}+$ cells. In addition, several studies have shown that MnSOD may act as a novel tumor suppressor gene and that overexpression of MnSOD can significantly decrease tumor promotion and key oncogenic survival and pro-inflammatory signaling pathways such as activator protein 1 (AP-1) and NF- $\kappa \mathrm{B}$ [19-21]. Therefore, regulating the intracellular redox status has the potential to augment the effects of current chemotherapeutic treatments and also block survival and pro-inflammatory pathways in tumor promotion sensitive cancer cells.

On the contrary, several studies have suggested that knockdown of APE1/Ref-1 can lead to apoptosis. APE1 /Ref-1 is known to be induced by oxidative stress [22]. However, APE1/Ref-1 expression has been shown to be inversely correlated with apoptosis, suggesting an antiapoptotic function of the multifunctional protein [23]. Unnikrishnan and colleagues presented data that oxidative stress can alter the function of APE1/Ref- 1 and the apoptotic response was increased in APE/Ref-1 haploinsufficient mice [24]. Within this study heterozygous deletion of APE1/Ref-1 resulted in decreased NF- $\kappa$ B DNA binding activity, accompanied by increases in GADD45 gene expression, p53 stability and caspase activity [24]. Previous studies have also shown that loss of APE1 /Ref-1 resulted in increased TNF-induced apoptosis [25]. In addition, homozygous deletion of the APE1/Ref-1 gene is embryonic lethal, suggesting the importance of APE1/Ref-1 expression in cell survival. Therefore, modulating APE1/Ref-1 expression can play either a cell survival or apoptotic role in tumorigenesis, but yet identifies APE/Ref-1 as a key controller of intracellular redox changes in chemoprevention. Clinically, APE1/Ref-1 variants have been identified in the human population [26]; and these variants have been associated with increased cancer risk. Together, these data point toward an approach to improve drug target specificity and personalized medicine in the prevention and treatment of tumors with upregulated APE1/Ref-1 expression. Nevertheless, further studies are ongoing to determine whether the significant decrease seen in the colony-forming ability of JB6 P+ cells was due to apoptosis or changes in mitochondrial function. So far, we have not found any associated toxicity following siRNA transfection and no indications of an apoptotic phenotype in cells that underwent transfection. Moreover, we will continue to assess the role of APE1/Ref-1 redox activity in early tumor promontion by utilizing known antioxidant-inducing natural products to determine whether antioxidant expression can modulate the redox functions of APE1/Ref- 1 in early stage skin carcinogenesis. In conclusion, our results suggest that Ref-1 promotes early tumorigenesisby via contributing to NF- $\kappa \mathrm{B}$ activation and the pro-inflammatory response. Therefore, Ref- 1 might be able to serve as a novel target for chemoprevention.

\section{REFERENCES}

[1] L. A. Loeb and B. D. Preston, "Mutagenesis by Apurinic/Apyrimidinic Sites," Annual Review of Genetics, Vol. 20, 1986, pp. 201-230. doi:10.1146/annurev.ge.20.120186.001221

[2] R. M. Schaaper, T. A. Kunkel and L. A. Loeb, "Infidelity of DNA Synthesis Associated with Bypass of Apurinic Sites," Proceedings of the National Academy of Sciences of the United States, Vol. 80, No. 2, 1983, pp. 487-491. doi:10.1073/pnas.80.2.487

[3] D. M. Wilson and D. Barsky, "The Major Human Abasic Endonuclease: Formation, Consequences and Repair of Abasic Lesions in DNA," Mutation Research, Vol. 485, No. 4, 2001, pp. 283-307. doi:10.1016/S0921-8777(01)00063-5

[4] A. R. Evans, M. Limp-Foster and M. R. Kelley, "Going APE over Ref-1," Mutation Research, Vol. 461, No. 2, 2000, pp. 83-108. doi:10.1016/S0921-8777(00)00046-X

[5] B. Muller-Hubenthal, M. Azemar, D. Lorenzen, M. Huber, M. A. Freudenberg, et al., "Tumor Biology: TumourAssociated Inflammation versus Antitumor Immunity," Anticancer Research, Vol. 29, No. 11, 2009, pp. 47954806.

[6] M. H. Han, W. K. Yon, H. Lee, et al., “Topical Application of Silymarin Reduces Chemical-Induced Irritant Contact Dermatitis in BALB/c Mice," International Immunopharmacology, Vol. 7, No. 13, 2007, pp. 1651-1658. doi:10.1016/j.intimp.2007.08.019

[7] P. C. Taylor, A. M. Peters, E. Paleolog, et al., "Reduction of Chemokine Levels and Leukocyte Traffic to Joints by Tumor Necrosis Factor Alpha Blockade in Patients with Rheumatoid Arthritis," Arthritis \& Rheumatism, Vol. 43, No. 1, 2000, pp. 38-47.

doi:10.1002/1529-0131(200001)43:1<38::AID-ANR6>3. 0.CO;2-L

[8] E. Van Coillie, J. Van Damme and G. Opdenakker, "The MCP/Eotaxin Subfamily of CC Chemokines," Cytokine Growth Factor Reviews, Vol. 10, No. 1, 1999, pp. 61-86. doi:10.1016/S1359-6101(99)00005-2

[9] M. Tucci, C. Quatraro, M. A. Frassanito and F. Silvestris, "Deregulated Expression of Monocyte Chemoattractant Protein-1 (MCP-1) in Arterial Hypertension: Role of Endothelial Inflammation and Atheromasia," Journal of Hypertension, Vol. 24, 2006, pp. 1307-1318. doi:10.1097/01.hjh.0000234111.31239.c3

[10] M. O’Hayre, C. L. Salanga, T. M. Handel and S. J. Allen, "Chemokines and Cancer: Migration, Intracellular Signaling and Intercellular Communication in the Microenvironment," Biochemical Journal, Vol. 409, No. 3, 2008, pp. 635-649. doi:10.1042/BJ20071493

[11] J. Zhang, L. Chen, M. Xiao, et al., "FSP1 ${ }^{+}$Fibroblasts Promote Skin Carcinogenesis by Maintaining MCP-1 Mediated Macrophage Infiltration and Chronic Inflammation," American Journal of Pathology, Vol. 178, No. 1, 2011, pp. 382-390. doi:10.1016/j.ajpath.2010.11.017

[12] A. Kapp, G. Zeck-Kapp, W. Czech and E. Schopf, “The Chemokine RANTES is More than a Chemoattractant: Characterization of Its Effect on Human Eosinophil Oxi- 
dative Metabolism and Morphology in Comparison with IL-5 and GM-CSF," Journal of Investigative Dermatology, Vol. 102, No. 6, 1994, pp. 906-914. doi:10.1111/1523-1747.ep12383399

[13] S. Kakolyris, L. Kaklamanis, A. Giatromanolaki, M. Koukourakis, S. I. D. Hickson, et al., "Expression and Subcellular Localization of Human AP Endonuclease 1 (HAP1/Ref-1) Protein: A Basis for Its Role in Human Disease," Histopath, Vol. 33, No. 6, 1998, pp. 561-569. doi:10.1046/j.1365-2559.1998.00541.x

[14] N. H. Colburn, B. F. Former, K. A. Nelson and S. H. Yuspa, "Tumour Promoter Induces Anchorage Independence Irreversibly," Nature, Vol. 281, 1979, pp. 589591. doi:10.1038/281589a0

[15] Y. Xu, D. H. Moore, J. Broshears, L. Liu, T. M. Wilson, et al., "The Apurinic/Apyrimidinic Endonuclease (APE/ Ref-1) DNA Repair Enzyme Is Elevated in Pre-Malignant and Malignant Cervical Cancer," Anticancer Research, Vol. 17, No. 5B, 1997, pp. 3713-3719.

[16] D. H. Moore, H. Michael, R. Tritt, S. H. Parsons and M. R. Kelley, "Alterations in the Expression of the DNA Repair/Redox Enzyme APE/Ref-1 in Epithelial Ovarian Cancers," Clinical Cancer Research, Vol. 6, No. 2, 2000, pp. 602-609.

[17] K. Ando, S. Hirao, Y. Kabe, Y. Ogura, I. Sato, Y. Yamaguchi, T. Wada and H. Handa, "A New APE1/Ref-1Dependent Pathway Leading to Reduction of NF-Kappab and AP-1, and Activation of Their DNA-Binding Activity," Nucleic Acids Research, Vol. 36, No. 13, 2008, pp. 4327-4336. doi:10.1093/nar/gkn416

[18] J. Liu, X. Gu, D. Robbins, G. Li, R. Shi, et al., "Protandim, a Fundamentally New Antioxidant Approach in Chemoprevention Using mouse Two-Stage Skin Carcinogenesis as a Model," PLoS One, Vol. 4, No. 4, 2009, p. e5284. doi:10.1371/journal.pone.0005284

[19] Y. Zhao, L. Chaiswing, T. D. Oberley, I. Batinic-Harberle, W. St. Clair, et al., "A Mechanism-Based Antioxidant Approach for the Reduction of Skin Carcinogenesis," Cancer Research, Vol. 65, No. 4, 2005, pp. 1401-1405. doi:10.1158/0008-5472.CAN-04-3334

[20] Y. Zhao, Y. Xue, T. D. Oberley, K. K. Kiningham, S. M. Lin, et al., "Overexpression of Manganese Superoxide Dismutase Suppresses Tumor Formation by Modulation of Activator Protein-1 Signaling in a Multistage Skin Carcinogenesis Model," Cancer Research, Vol. 61, No. 16, 2001, pp. 6082-6088.

[21] Y. Zhao, K. K. Kiningham, S. M. Lin and D. K. St. Clair, "Overexpression of MnSOD Protects Murine Fibrosarcoma Cells (FSa-II) from Apoptosis and Promotes a Dif- ferentiation Program upon Treatment with 5-Azacytidine: Involvement of MAPK and NFkappaB Pathways," Antioxidants \& Redox Signaling, Vol. 3, No. 3, 2001, pp. 375-386. doi:10.1089/15230860152409022

[22] S. Grosch, G. Fritz and B. Kaina, "Apurinic Endonuclease (Ref-1) Is Induced in Mammalian Cells by Oxidative Stress and Is Involved in Clastogenic Adaptation," Cancer Research, Vol. 58, No. 19, 1998, pp. 4410-4416.

[23] C. V. Ramana, I. Boldogh, T. Izumi and S. Mitra, “Activation of Apurinic/Apyrimidinic Endonuclease in $\mathrm{Hu}-$ man Cells by Reactive Oxygen Species and Its Correlation with Their Adaptive Response to Genotoxicity of Free Radicals," Proceedings of the National Academy of Sciences of United States, Vol. 95, No. 9, 1998, pp. 50615066. doi:10.1073/pnas.95.9.5061

[24] A. Unnikrishnan, J. J. Raffoul, H. V. Patel, T. M. Prychitko, N. Anyangwe, et al., "Oxidative Stress Alters Base Excision Repair Pathway and Increases Apoptotic Response in Apurinic/Apyrimidinic Endonuclease 1/Redox Factor-1 Haploinsufficient Mice," Free Radical Biology \& Medicine, Vol. 46, No. 11, 2009, pp. 1488-1499.

[25] J. L. Hall, X. Wang, V. Adamson, Y. Zhao and G. H. Gibbons, "Overexpression of Ref-1 Inhibits Hypoxia and Tumor Necrosis Factor-Induced Endothelial Cell Apoptosis through Nuclear Factor- $\kappa$ B-Independent and -Dependent Pathways," Circulation Research, Vol. 88, 2001, pp. 1247-1253. doi:10.1161/hh1201.091796

[26] M. Z. Hadi, M. A. Coleman, K. Fidelis, H. W. Mohrenweiser and D. M. Wilson III, "Functional characterization of Ape1 variants identified in the human population," Nucleic Acids Research, Vol. 28, No. 20, 2000, pp. 38713879. doi:10.1093/nar/28.20.3871

\footnotetext{
Abbreviations

AP, Apurinic/apyrimidinic; AP-1, activator protein 1; APE1, apurinic (apyrimidinic) endonuclease 1; DMSO, dimethyl sulfoxide; FBS, fetal bovine serum; MCP-1, monocyte chemotactic protein-1; MnSOD, manganese superoxide dismutase; NF- $\kappa \mathrm{B}$, nuclear factor kappa $\mathrm{B}$; PBS, phosphate buffered saline; Ref-1, redox factor-1; ROS, reactive oxygen species; SDHB, succinate dehydrogenase subunit B; siRNA, small interfering RNA; TNF, tumor necrosis factor; TPA, 12-O-tetradecanoylphorbol-13-aceteate.
} 